\title{
Quantification of magnetic force microscopy images using combined electrostatic and magnetostatic imaging
}

\author{
R. D. Gomez, ${ }^{\text {a) }}$ A. O. Pak, A. J. Anderson, E. R. Burke, A. J. Leyendecker, \\ and I. D. Mayergoyz \\ Department of Electrical Engineering and Laboratory for Physical Sciences, University of Maryland, \\ College Park, Maryland 20742
}

\begin{abstract}
A method for calibrating the force gradients and probe magnetic moment in phase-contrast magnetic force microscopy (MFM) is introduced. It is based upon the combined electrostatic force microscopy EFM and MFM images of a conducting non magnetic metal strip. The behavior of the phase contrast in EFM is analyzed and modeled as a finite area capacitor. This model is used in conjunction with the imaging data to derive the proportionality constant between the phase and the force gradient. This calibration is further used to relate the measured MFM images with the field gradient from the same conducting strip to derive the effective magnetic moment of the probe. The knowledge of the phase-force gradient proportionality constant and the probe's effective moment is essential to directly quantify field derivatives in MFM images. (C) 1998 American Institute of Physics. [S0021-8979(98)27711-5]
\end{abstract}

\section{INTRODUCTION}

Magnetic force microscopy (MFM) has become a standard diagnostic workhorse in understanding surface magnetism. In its basic implementation, the technique maps an image which is proportional to the local magnetostatic force gradient between a ferromagnetic sample and a magnetic probe. In an ideal case of a magnetic dipole probe, the force $F^{m}$ is the gradient of the magnetostatic energy, $(\bar{m} \cdot \bar{B})$, and the force gradient can be expressed as, ${ }^{1}$

$$
\frac{\partial F^{m}(x, y)}{\partial z}=\sum_{i=1}^{3} \mu_{0} m_{i} \cdot \frac{\partial^{2} H_{i}(x, y)}{\partial z^{2}} .
$$

The image depends upon the direction of the probe's magnetic moment, and contains the contribution of the different components of the surface stray field. In practice, it is customary to premagnetize the probe along the surface normal direction, $\hat{z}$, which makes the contrast proportional to the second derivative of the normal magnetic field component. By using Eq. (1), it is possible to interpret the images and extract the values of some parameters, such as the transition lengths and zigzag deviation of recorded patterns, the width of the domain wall, and the direction of local surface magnetization of ferromagnetic surfaces, as well as other quantities that are dependent only on the spatial coordinates but are independent of the absolute magnitude of the interaction force. ${ }^{2}$ The difficulty in establishing the absolute values of the interaction force arises since the proportionality constant, $K_{p}$, between the measured oscillation phase, $\Delta \phi$ and the force gradient,

$$
\Delta \phi=K_{p} \cdot \frac{\partial F_{z}}{\partial z}
$$

is dependent upon the specific mechanical characteristics of the probe and its environment, and is generally unknown. In addition, the probe's effective magnetic moment, $\bar{m}$ appear-

\footnotetext{
${ }^{a)}$ Electronic mail: rdgomez@eng.umd.edu
}

ing in Eq. (1) is also undetermined. Thus, without the knowledge of these two important probe-dependent parameters, Eq. (1) and Eq. (2) can only be taken as qualitative descriptions of the imaging contrast.

Several research groups have attempted to calibrate the probe and provide estimates for tip-sample interaction force. Unfortunately, due to space constraints in this article, we refer the reader to the literature. Previous approaches have involved the imaging of a standardized system, such as a metal strip $^{3,4}$ or single-crystal surfaces ${ }^{5}$ or the usage of sophisticated methods to measure the magnetic moment of the probe and compare the acquired data with various models for the probe. ${ }^{6,7}$ There are, however, no methods that prescribe a self-contained calibration procedure of both the probe's mechanical and magnetic characteristics by utilizing only the measurements of the instrument itself. In this work, we propose a straightforward method for estimating $K_{p}$ and $m_{z}$, thereby allowing a direct quantification of the MFM response. The basic concept uses the equivalence of the electrostatic (EFM) and magnetic interactions in generating the force gradients on a conducting metal strip. This was accomplished by imaging a test sample, an $11 \mu \mathrm{m}$ wide Au metal line on silicon, using EFM and MFM with the same probe.

\section{EXPERIMENTAL RESULTS AND DISCUSSION}

The relationship between the force gradient and phase in Eq. (2) with constant $K_{p}$ is valid as long as the scanned probe microscope (SPM) operates in a linear regime, which is tacitly assumed in our analysis. The force, $F$, could be due to either electrostatic or magnetostatic interactions. To determine $K_{p}$, we obtained an EFM image of the test sample by biasing the metal structure at voltage $V$ relative to the probe at ground and examined the dependence of the phase contrast versus bias voltage and height. Note that a regular MFM probe was used for this experiment, since the magnetic coating is also electrically conducting. A typical EFM image is shown in Fig. 1 (right), along with a representative cross sectional profile. The electrostatic forces are purely attractive 


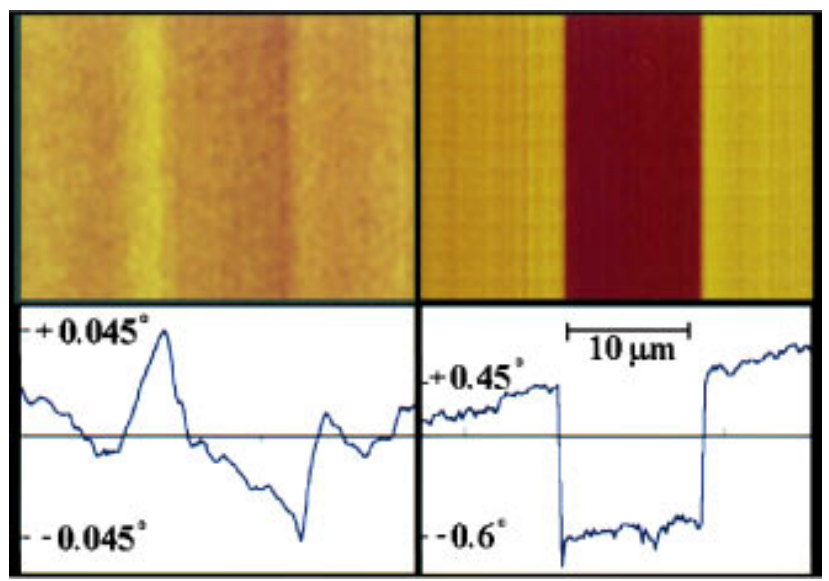

FIG. 1. Right: EFM image of a conducting $11 \mu \mathrm{m}$ wide metal strip at $V$ $=2 \mathrm{~V}$. Left: MFM image of the same strip with $11 \mathrm{~mA}$ current. Bottom: Average line profiles across the strip.

so that the EFM contrast appears dark in the regions where the energized metal structure is present. The electrostatic force is a function of the bias voltage, $V$, as well as its capacitance:

$$
F_{z}^{e}=-\frac{V^{2}}{2} \frac{\partial C}{\partial z},
$$

and the force gradient appearing in Eq. (2) can be expressed as

$$
\frac{\partial F_{z}^{e}(x, y)}{\partial z}=-\frac{V^{2}}{2} \frac{\partial^{2} C(x, y)}{\partial z^{2}} .
$$

Equation (4) shows that the electrostatic force gradients and consequently the EFM images should vary as $V^{2}$ and its curvature and spatial variations are dependent only on the capacitance. This is experimentally verified in Fig. 2, which shows the acquired images at different voltages and at a constant height. The contrast has been reversed using software in order to emphasize the changing magnitude of the force gradient. In this experiment, the same area of the sample was continuously scanned at a tip height of $50 \mathrm{~nm}$, as the bias voltage was progressively incremented by $100 \mathrm{mV}$. The values of the maximum phase contrast in the range from 100 $\mathrm{mV}$ to $2 \mathrm{~V}$ is shown at the bottom of Fig. 2. The fit to a quadratic function convincingly shows the $V^{2}$ dependence. As mentioned previously, all of the spatial variation in the electrostatic contrast is contained in the capacitance term so that the coefficient of the quadratic term in the fit is proportional to the second derivative of the capacitance with respect to $z$. Furthermore, since there are no dielectric materials in the gap region, the capacitance of the system depends only upon the geometrical arrangement.

To model the capacitance of the system, we considered the dependence of the image on the separation or the lift height, $h$, between the sample and probe. Figure 3 shows the variation of the image contrast at constant voltage, $V=1.8 \mathrm{~V}$, as a function of the height, $h$. As in Fig. 2, the same region was imaged repeatedly, as the lift height was incremented to the labeled values. The glitch separating each increment is an instrumental artifact and arises due to the finite response time

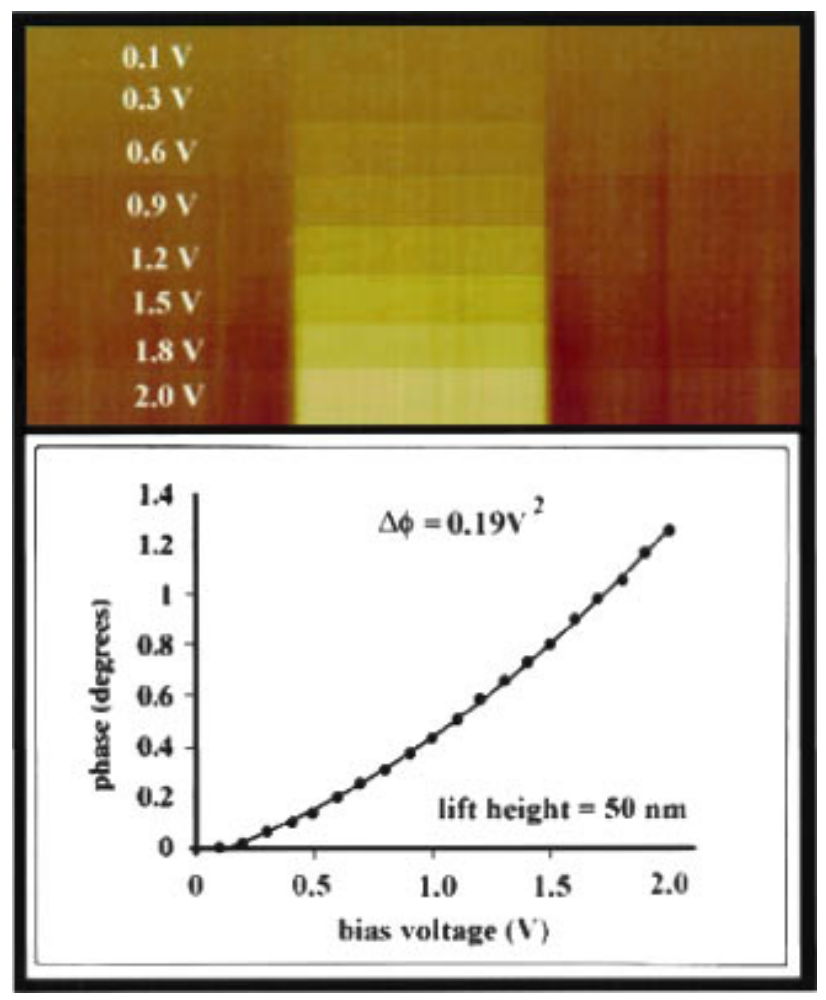

FIG. 2. EFM contrast dependence on bias voltage. Top: EFM image at a lift height of $50 \mathrm{~nm}$ with increasing voltage. Bottom: Plot of the maximum contrast as a function of voltage, fitted to a quadratic function $\Delta \phi$ $=0.19 \mathrm{~V}^{2}$.

of the feedback system. Nevertheless, the log-log plot of the maximum phase contrast versus $h$ at the bottom of the image clearly shows the strong dependence of the phase image on $h$. The lines are various power law curves, and the best fit to the data is $\Delta \phi \sim h^{-1.9}$. The nearly $h^{-2}$ dependence suggests that the interaction between the sample and probe can be modeled as a finite area parallel plate capacitor.

\section{ANALYSIS}

The capacitance for a square parallel plate capacitor can be solved using the Schwarz transformation technique ${ }^{8}$ and the second derivative with respect to the separation distance, $h$, is given by

$$
\frac{\partial^{2} C}{\partial h^{2}}=2 \epsilon_{0} A \times\left(\frac{1}{h^{3}}+\frac{2}{\pi^{3 / 2} R h^{2}}\right),
$$

where $A$ is the effective surface area and $R$ is the tip radius. In order to relate Eq. (5) with the parameters of the instrument, we assume that the apex of the pyramidal probe can be approximated by a square cross section of width $\sqrt{\pi} R$. The first term in Eq. (5) is identified with an infinite plate capacitor $(R \gg h)$, and the second term is due to the fringing effects of the field at the edges. It is clear that as the sample-probe separation, $h$ tends to zero, Eq. (5) is dominated by the first term. However, the relative contribution of the fringe effects increases as $0.36(h / R)$, so that the fringing field contributes about $36 \%$ for $R=h$ and significantly more for $h \gg R$. This is precisely the reason why the phase contrast in Fig. 3 follows the $h^{-2}$ power law rather than $h^{-3}$. Substituting Eq. (5) into 


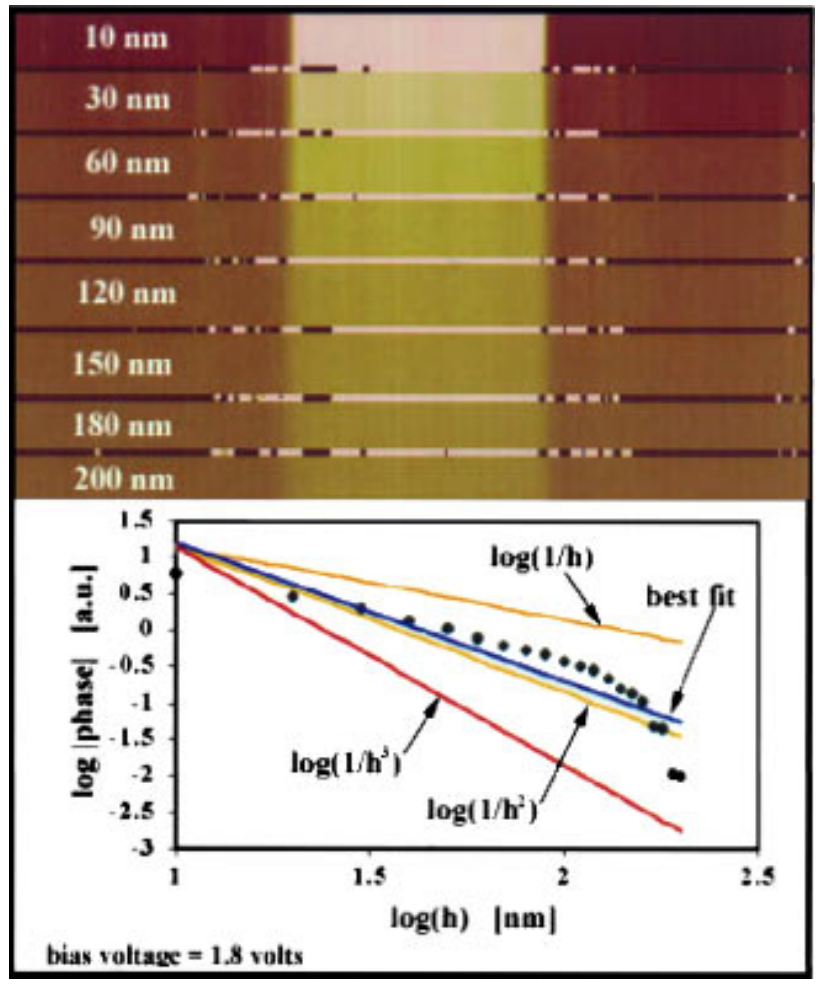

FIG. 3. EFM contrast dependence on lift height. Top: EFM image at a constant bias voltage of $1.8 \mathrm{~V}$ at varying lift heights. Bottom: Log-log plot of the maximum contrast as a function of height. Fit corresponds to $\log \mid$ phase $\mid=-1.9 \log (h)+3.1 ;$ plots for $\log (1 / h), \log \left(1 / h^{2}\right)$, and $\log \left(1 / h^{3}\right)$ are also shown for comparison.

Eq. (4), and using the result into Eq. (2), yields the explicit expression for the calibration for the parameter, $K_{p}$.

$$
K_{p}=2\left(\frac{|\Delta \phi|}{V^{2}}\right)_{\text {meas }} / 2 \epsilon_{0} A \times\left(\frac{1}{h^{3}}+\frac{2}{\pi^{3 / 2} R h^{2}}\right),
$$

where the numerator is the coefficient of the quadratic term of the data in Fig. 2, and the denominator is calculated directly from the parameters: $R=40 \mathrm{~nm}$, related to the effective tip curvature, and the lift height, $h=50 \mathrm{~nm}$. Inserting these numbers into Eq. (6) yields $K_{p}=369 \mathrm{degs} /$ Newtons $/ \mathrm{m}$. Using this derived calibration constant, we can then calculate the effective probe moment along the $z$ direction. In this case, we assume for convenience that the probe is nominally oriented along the $z$ axis, so that only the $z$ component of the surface stray fields contribute to the image. Again, we can make use of Eq. (1) and Eq. (2) to show that

$$
m_{z}=\frac{\partial F^{m}}{\partial z} / \frac{\partial^{2} B_{z}}{\partial z^{2}}=|\Delta \phi|_{\text {meas }} / K_{p} \frac{\partial^{2} B_{z}}{\partial z^{2}} .
$$

We now image the same current strip at a specific current, and measure the change in phase. A representative image is shown in Fig. 1(left), where the bright and dark contrasts are most pronounced at the edges of the strip as expected for the maximum normal field component. The field from this nonferromagnetic current strip is well known and the field derivatives in Eq. (7) at a distance of $h=50 \mathrm{~nm}$ and at a current of $11 \mathrm{~mA}$ is $5.5 \times 10^{10} \mathrm{~T} / \mathrm{m}^{2}$. Using this value with the maxi- mum measured phase of 0.045 degrees in Fig. 1 (left), we obtain the effective moment for the probe, $m_{z}=2.22$ $\times 10^{-15} \mathrm{~A} \mathrm{~m}^{2}\left(2.22 \times 10^{-12} \mathrm{emu}\right)$. This value is consistent with previous estimates, ${ }^{4,6}$ albeit somewhat lower, which could be attributed to differences on the specific probes used. As a plausibility check, we can compute the magnetization of the probe by dividing $m_{z}$ with effective magnetic volume. If the effective volume is assumed to be that of a half-sphere $\left(2 \pi / 3 R^{3}\right.$ ) with $R=40 \mathrm{~nm}$ (film thickness at $40 \mathrm{~nm}$ as well) then the magnetization is in excess of $18000 \mathrm{emu} / \mathrm{cc}$, which is much larger than the $400-800 \mathrm{emu} / \mathrm{cm}^{3}$ remanent magnetization of the $\mathrm{CoCr}$ thin film coating. However, as pointed out by previous authors, ${ }^{9}$ the actual magnetic volume can be considerably larger than the volume of the half sphere shell at the apex of tip. In this particular case, if the effective volume were to include magnetic material up to a distance of $200 \mathrm{~nm}$ from the apex, then we obtain $M_{p}=586 \mathrm{emu} / \mathrm{cc}$ which is closer to the expected saturation magnetization of the $\mathrm{CoCr}$ coating.

In conclusion, the value of this calibration method is that the procedure is self-contained, and that all calibrations are derivable from the measurements themselves. It does not necessitate other sophisticated external measurements nor assumptions about the specific mechanical properties of the system. This will save considerable time and effort, and avoid the ever present doubt of whether the externally measured probe characteristics are invariant under actual operation. The procedure outlined in this paper, however, should be considered as an initial step that could be improved considerably. One area for improvement is the replacement of the analytical expression for the finite square area capacitor model with a more accurate numerical calculation involving the actual geometry of the pyramidal apex. This must be accompanied by a deconvolution procedure that takes into account the vertical excursion of the oscillating in the heightdependence measurements. Finally, it should be pointed out that while the calibration procedure here is carried out for phase-detection, a similar procedure can be performed for frequency or amplitude modes of force gradient mapping.

This work was partially supported by NSF MRSEC and ARO Physics Contract No. 36114 PH-RIP. We thank Professor R. Webb for the samples used.

${ }^{1}$ P. Grutter, H. J. Mamin, and D. Rugar, in Scanning Tunneling Microscopy Vol. II, edited by R. Wiesendanger and H.-J. Guntherodt (Springer, Berlin, 1992), p. 151.

${ }^{2}$ R. D. Gomez, E. R. Burke, and I. D. Mayergoyz, J. Appl. Phys. 79, 6441 (1996).

${ }^{3}$ T. Goddenhenrich, H. Lemke, M. Muck, U. Hartmann, and C. Heiden, Appl. Phys. Lett. 57, 2612 (1990).

${ }^{4}$ K. Babcock, V. Elings, J. Shi, D. D. Awshalom, and M. Dugas, Appl. Phys. Lett. 69, 705 (1996).

${ }^{5}$ S. Huo, J. E. Bishop, J. W. Tucker, W. M. Rainforth, and H. A. Davies, IEEE Trans. Magn. 33, 4056 (1997).

${ }^{6}$ G. P. Heydon, A. N. Farley, S. R. Moon, M. S. Valera, and S. L. Tomlinson, IEEE Trans. Magn. 33, 4059 (1997).

${ }^{7}$ R. Proksch, G. D. Skidmorem, E. D. Dahlberg, S. Foss, J. J. Schmidt, C. Merton, B. Walsh, and M. Dugas, Appl. Phys. Lett. 69, 2599 (1996).

${ }^{8}$ See, for example, R. S. Elliot, Electromagnetics (McGraw-Hill, New York, 1996), p. 180.

${ }^{9}$ K. Babcock, M. Dugas, V. Elings, and S. Loper, IEEE Trans. Magn. 30, 4503 (1994). 